\title{
La composition microélectrophorétique des micelles de la caséine du lait de brebis de diamètres différents
}

\author{
par \\ Dr Jovan DJORDJEVIC* et Mr Marijana CARIC**
}

La découverte de la caséine $\chi$ et la mise au point de son rôle protecteur et stabilisateur du complexe total du phosphocaséinate de chaux représentaient une stimulation de plus pour des études nouvelles de ce protéide, dont les propriétés dominent de nombreux caractères technologiques du lait. Ces découvertes donnaient l'espoir qu'on pourrait aller plus vite à résoudre l'essence de la structure des globules de la caséine.

Hostettler et Imhof [11], Davidov et al. [6] et autres auteurs ont constaté la polydispersité des globules de la caséine et ont déterminé certains facteurs qui influencent la grandeur de ces particules. Ces faits ont amené le problème de la composition des globules de diamètres différents. Les recherches concernant ces questions ont donné des réponses contradictoires sans égard à la méthode employée. C'est dire que les résultats étaient différents quand on utilisait soit le rapport $\mathrm{Ca} /$ azote de la caséine, soit le rapport phosphore organique / azote de la caséine, soit la composition électrophorétique des particules de la caséine de diamètres différents.

Webb et Johnson [16] citent les résultats de Heyndrickx et Vleeschauwer qui nous suggèrent en conclusion que la composition électrophorétique des petites micelles est différente de celle de la caséine du lait d'origine. Annibaldi [1] a constaté aussi qu'il existe des différences de composition électrophorétique entre les particules de la caséine de diamètres différents. Cela fait que les petits globules contiennent plus de caséine $\alpha$ et moins de caséine $\beta$ que les

\footnotetext{
* Professeur à la Faculté d'Agriculture, Zemun (Yougoslavie).

** Assistant à la Faculté de Technologie, Novi Sad.
} 
micelles de diamètre plus grand. Grâce à ce fait, les particules de diamètre petit et moyen sont plus sensibles à l'action de la chymosine. Snow et Hay [13] annoncent que la caséine n'a pas une composition constante dans les micelles de grandeur différente et que la caséine $\alpha, \beta$ et $\chi$ n'ont pas la même corrélation dans toutes les particules. Bohren et Wenner [4] ont établi que le rapport entre le phosphore organique et l'azote dans les petits globules de caséine est sensiblement inférieur à celui de la caséine totale.

Au contraire, Ford et al. [7] n'ont pas établi de différences, excepté les plus grandes particules qui contiennent moins de phosphore organique. Des résultats de Wake et Baldwin [14] on peut voir que les particules de grandeur différente ont la même composition électrophorétique.

Les résultats cités montrent qu'il y a des désaccords relatifs à la composition des micelles de caséine de diamètre différent et qu'il est nécessaire d'obtenir de nouvelles preuves pour répondre à cette question. On accorde une grande importance à ce problème car il nous mène indirectement à l'essence de la structure du complexe phosphocaséinate de chaux.

Les recherches concernant ces questions ont été effectuées presque exclusivement sur le lait de vache tandis que les résultats concernant les propriétés et la composition électrophorétique de la caséine du lait de brebis sont très rares ou inexistants. Pour cette raison on a décidé d'étudier plus précisément le lait de brebis en posant l'accent sur l'examen de la caséine. Dans ce travail nous présenterons les résultats sur la composition électrophorétique des particules de caséine de diamètres différents.

\section{Matériel et méthodes}

Chaque échantillon était composé du lait de 5 brebis de la race tzigaia. La séparation de la matière grasse était effectuée à l'aide d'une écrémeuse qui éliminait 99 p. 100 des lipides du lait.

Séparation des micelles de caséine de diamètres différents. Pour séparer les micelles de diamètres différents on a utilisé 1'ultracentrifugation différentielle. On s'est servi de trois forces différentes : $10300 \mathrm{~g}, 47600 \mathrm{~g}$ et $144400 \mathrm{~g}$ (respectivement : $10000-21500$ et 38000 tours $/ \mathrm{mn}$ ). En utilisant ces forces on a pu séparer trois groupes de particules : celles qui dépassent $140,7 \mathrm{~m} \mu$, celles dont le diamètre se trouve entre 65,5 et $140,7 \mathrm{~m} \mu$ et celles inférieures à $65,5 \mathrm{~m} \mu$.

Préparation des solutions de caséine pour la microélectrophorèse. Les sédiments obtenus par ultra-centrifugation ont été reconstitués et additionnés de $40 \mathrm{ml}$ d'eau distillée. Puis la solution a été amenée au point isoélectrique de la caséine à l'aide du tampon 
d'acétate. Après précipitation de la protéine on a décanté le liquide, on a rincé le sédiment plusieurs fois avec de l'eau distillée, puis on l'a dissout dans l'urée 7M.

La dialyse a été effectuée avec un volume de solution tampon dix fois plus grand que celui de l'échantillon. La dialyse avait lieu pendant $24 \mathrm{~h}$ à $2-4^{\circ} \mathrm{C}$, sans changement de la solution tampon [8].

Microélectrophorèse. Pour la microélectrophorèse on s'est servi de la technique "moving boundary » préconisée par Tiselius [2], sur un appareil de la maison Kern. On a utilisé le tampon véronal à $\mathrm{pH} 8,6$ avec un pouvoir ionique $\mu=0,1$ [3].

\section{Résultats et discussion}

On a utilisé la microélectrophorèse pour obtenir des informations sur la composition des particules de caséine de diamètres différents et pour déterminer les rapports entre les caséines $\alpha \mathrm{s}, \chi, \beta$ et $\gamma$.

Le graphique 1 représente la photographie d'un échantillon des particules de caséine dépassant $140.7 \mathrm{~m} \mu$ à la microélectrophorèse avec interprétation des résultats. Pour l'observation et l'enregistrement photographique des bornes des fractions on a utilisé le système optique préconisé par Tiselius, qu'on connaît dans la littérature comme * Schlieren Band » $[2,5]$.

Les résultats de la microélectrophorèse effectuée avec les sédiments de la caséine, de même que les résultats qui concernent la caséine complète, sont présentés dans le tableau 1. Le terme " caséine complète " signifie que l'échantillon n'a pas été soumis à l'ultra-centrifugation et qu'il représente un mélange naturel des particules de caséine de diamètres différents.

Ces résultats révèlent que la caséine $\alpha$ constitue 57,7 à 66,7 p. 100 (moyenne 63,6 p. 100) des particules dont le diamètre dépasse $140,7 \mathrm{~m} \mu$; les particules dont le diamètre varie de 65,5 à $140,7 \mathrm{~m} \mu$ contiennent de 61,4 à 69,0 p. 100 (moyenne 64,0 p. 100) de caséine $\alpha$; dans le groupe des particules inférieures à $65,5 \mathrm{~m} \mu$ la caséine $\alpha$ fait 57,0 à 70,6 p. 100 (moyenne 64,0 p. 100). L'intervalle de variation de la caséine $\alpha$ dans l'échantillon complet est de 61,0 à 69,2 p. 100 avec une moyenne de 65,2 p. 100 . De là, il est évident que les moyennes qui représentent la participation de la caséine $\alpha$ dans les globules de diamètres différents sont d'accord avec les données relatives à l'échantillon complet.

La caséine $\chi$ fait 18,9 p. 100 de la caséine $\alpha$ dans le groupe des particules qui dépassent $140,7 \mathrm{~m} \mu$; elle constitue 18,5 p. 100 de la caséine $\alpha$ dans les particules de grandeur moyenne; elle fait 19,5 p. 100 de la caséine $\alpha$ dans les petites micelles et 20,4 p. 100 dans la caséine complète. 

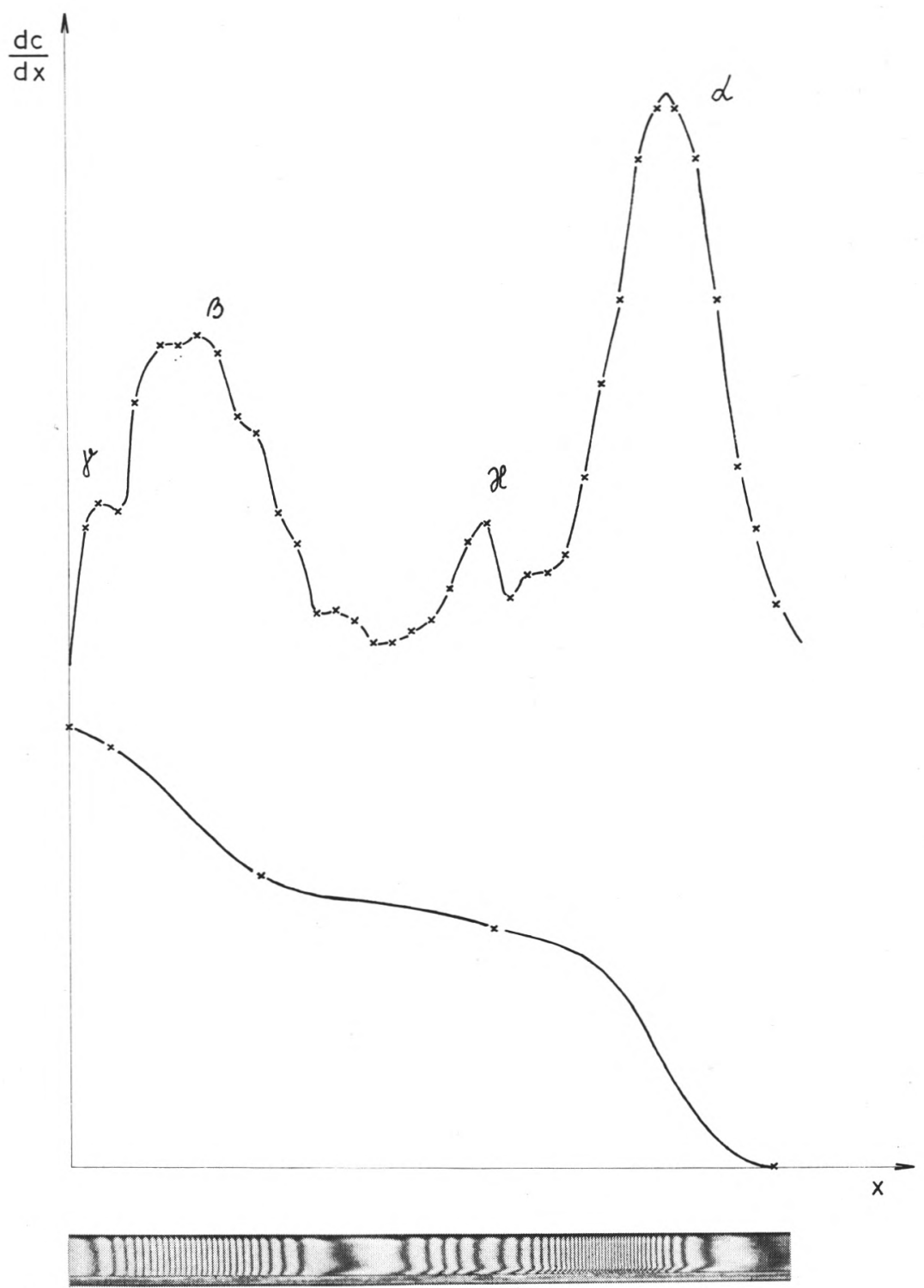

Le mouvement de la caséine dans le courant électrique

fig. 1

Une des analyses microélectrophorétiques des particules de la caséine dépassant 140,7 $\mathrm{m} \mu$ 
TABLEAU 1. - Composition microélectrophorétique des particules de la caséine de diamètres différents

\begin{tabular}{|c|c|c|c|c|c|c|c|c|c|c|c|c|c|c|c|c|}
\hline \multirow{2}{*}{$\mathrm{N}^{\circ}$} & \multicolumn{4}{|c|}{$\varnothing>140.7 \mathrm{~m} \mu$} & \multicolumn{4}{|c|}{$65.5<\varnothing<140.7 \mathrm{~m} \mu$} & \multicolumn{4}{|c|}{$\varnothing<65.5 \mathrm{~m} \mu$} & \multicolumn{4}{|c|}{ Echantillon complet } \\
\hline & $\alpha_{\mathrm{S}}$ & $x$ & $\beta$ & $\gamma$ & $\alpha_{\mathrm{s}}$ & $\chi$ & $\beta$ & $\gamma$ & $\alpha_{\mathrm{s}}$ & $\chi$ & $\beta$ & $\gamma$ & $\alpha_{\mathrm{s}}$ & $\chi$ & $\beta$ & $\gamma$ \\
\hline 1 & 43.7 & 14.0 & 37.0 & 4.8 & 44.8 & 16.6 & 34.7 & 3.9 & 45.0 & 14.2 & 37.8 & 3.0 & 44.5 & 18.0 & 34.2 & 3.3 \\
\hline 2 & 50.6 & 9.4 & 36.8 & 3.2 & 51.0 & 11.7 & 33.7 & 3.6 & 46.7 & 10.3 & 40.0 & 3.0 & 48.0 & 13.0 & 35.9 & 3.1 \\
\hline 3 & 56.0 & 9.6 & 31.1 & 3.3 & 55.7 & 10.4 & 30.5 & 3.4 & 55.9 & 10.8 & 30.5 & 3.8 & 55.5 & 11.2 & 30.8 & 3.5 \\
\hline 4 & 53.0 & 11.2 & 31.8 & 4.0 & 53.2 & 11.1 & 32.9 & 2.8 & 54.3 & 12.0 & 30.0 & 3.7 & 53.9 & 12.8 & 29.9 & 3.4 \\
\hline 5 & 53.9 & 12.8 & 29.3 & 4.0 & 56.5 & 12.5 & 26.8 & 4.2 & 55.5 & 15.1 & 26.4 & 3.0 & 57.6 & 11.6 & 26.3 & 4.3 \\
\hline $\bar{x}$ & 51.6 & 12.0 & 32.5 & 3.9 & 52.2 & 11.8 & 32.5 & 3.5 & 51.5 & 12.5 & 32.7 & 3.3 & 51.9 & 13.3 & 31.3 & 3.5 \\
\hline
\end{tabular}

(Les quantités des caséines $\alpha_{\mathrm{s}}, \gamma, \beta$ et $\gamma$ sont exprimées comme les p. 100 de la caséine totale). 
La participation de la caséine $\beta$ dans les plus grandes particules varie de 29,3 p. 100 à 37,0 p. 100 (moyenne 32,5 ) ; les particules de grandeur moyenne contiennent de 26,8 à 34,7 p. 100 (moyenne 32,5) de la caséine $\beta$; cette caséine constitue de 26,4 à 40,0 p. 100 (moyenne $32,7)$ des plus petites particules. Les particules de la caséine complète contiennent de 26,3 à 35,9 p. 100 (moyenne 31,3) de la caséine $\beta$. Ces résultats montrent que les trois groupes de particules ne diffèrent pas sensiblement en ce qui concerne le contenu de la caséine $\beta$ et qu'il n'existe pas de corrélation directe entre la grandeur des micelles et leur contenu en caséine $\beta$.

La participation de la caséine $\gamma$ est différente selon les catégories des particules. C'est le seul composant électrophorétique dont la participation change régulièrement avec l'augmentation du diamètre des particules. C'est-à-dire, les particules les plus petites contiennent en apparence le moins (3,3 p. 100) et les micelles les plus grandes le plus $(3,9$ p. 100) de la caséine $\gamma$. Ces différences ne sont pas grandes en valeur absolue mais elles font 18 p. 100 du contenu de la caséine $\gamma$. Ces déviations pourraient être en conséquence des difficultés apparaissant lors de l'identification précise de la fraction qui se meut le plus lentement. Il faut toutefois signaler que les valeurs qu'on a obtenues pour la caséine $\gamma$ se trouvent dans les limites citées dans la littérature $[9,10]$.

Nos résultats montrent que la composition électrophorétique de la caséine du lait de brebis n'est pas fonction du diamètre des particules. Ils nous assurent aussi que les causes des variations qui apparaissent dans les analyses séparées devraient être cherchées ailleurs.

Nos données sont en accord avec celles de Waugh [15] qui a constaté qu'en mélangeant la caséine $\alpha_{\mathbb{S}}$ et $\chi$ on obtient immédiatement des complexes $\alpha_{s-} \chi$ qui représentent des produits d'interaction stoïchiométrique de ses composants. De plus, le rapport entre la caséine $\alpha_{s}$ et $\chi$ pour le lait de brebis est semblable à celui que l'auteur cité [15] a trouvé pour le lait de vache. Dans nos expériences, le rapport $\alpha_{\mathrm{s}} / \chi$ pour la caséine complète est $3,9 / 1$, ce qui est très voisin de la corrélation établie par Waugh " $4 / 1$ ». Pour les particules de diamètres différents ce rapport était comme suit :
Diamètre des particules :
Rapport $\alpha_{\mathrm{s}} / \chi$ :
$>140,7 \mathrm{~m} \mu$
$4,3 / 1$
65,5 à $140,7 \mathrm{~m} \mu$
$4,4 / 1$
$<65,5 \mathrm{~m} \mu$
$4,1 / 1$

Il est évident qu'il existe des différences entre ces rapports mais ils ne montrent pas une tendance à changer régulièrement pour qu'on puisse conclure qu'ils dépendent des dimensions des particules de la caséine.

Le rapport entre la caséine $\alpha$ et $\beta$ dans les particules de diamètres différents est comme suit : 


$\begin{array}{ll}\text { Diamètre des particules : } & \text { Rapport } \alpha / \beta \text { : } \\ >140,7 \mathrm{~m} \mu & 1,96 / 1 \\ 65,5 \text { à } 140,7 \mathrm{~m} \mu & 1,97 / 1 \\ <65,5 \mathrm{~m} \mu & 1,92 / 1\end{array}$

Pour l'échantillon complet ce rapport est de 2,08/1. Comme on peut le voir, ces rapports sont assez stables et appuient notre point de vue à savoir que la composition électrophérique est indépendante du diamètre des particules de la caséine. A cet égard nos résultats sont en accord avec ceux de Ford et al. [7] qui considèrent que la constance de la composition électrophorétique confirme le point de vue que les grandes particules sont des agrégats de monomères. Par conséquent, le rapport entre la caséine $\alpha$ et $\beta$ dans le lait de brebis serait 2/1. Ce rapport est bien plus étroit que celui qu'on trouve dans la littérature pour la caséine du lait de vache : $4 / 1$.

Le rapport des caséines $\alpha: \beta: \gamma$ dans les particules de diamètres différents varie de $16: 8: 1$ à $20: 10: 1$. La caséine complète de même que la catégorie des particules avec des diamètres variant de 65,5 à $140,7 \mathrm{~m} \mu$ ont le rapport $18: 9: 1$. Nous estimons que ce dernier est le rapport réel des composants électrophorétiques et que les extrêmes résultent des variations de la caséine $\gamma$ qui a servi comme unité pour calculer le rapport (les causes des variations de la caséine $\gamma$ ont déjà été discutées). Il faut noter que ce rapport diffère de celui des composants électrophorétiques de la caséine du lait de vache [7].

Les résultats de Ford et al. [7] montrent que le rapport entre le phosphore organique et l'azote de la caséine est constant dans les particules de diamètres différents et indiquent que leur composition est invariable. Cependant, ces auteurs indiquent qu'il est possible que les caséines $\alpha, \beta$ et $\gamma$ existent séparément sous forme de particules avec même répartition des diamètres. Dans la littérature [12] on trouve aussi des paramètres thermodynamiques de polymérisation des caséines $\alpha s, \beta$ et $\chi$ jusqu'à la formation des pentamères. Cependant, les caséines forment aussi bien des copolymères entre eux-mêmes, et plusieurs faits nous assurent dans l'opinion que les caséines $\alpha$, $\beta$ et $\gamma$ se trouvent ensemble dans les mêmes particules.

\section{R és u m é}

Dans ce travail on a étudié la composition électrophorétique des particules de caséine de diamètres différents dans le lait de brebis. Les résultats révèlent que la grandeur des particules n'influencent pas la composition électrophorétique.

Le rapport entre $\alpha_{\mathrm{s}}$ et $\chi$ caséine pour le lait de brebis est le même que celui pour le lait de vache : 4/1, tandis que les rapports $\alpha: \beta$ et $\alpha: \beta: \gamma$ pour la caséine du lait de brebis sont différents de ceux qu'on trouve pour le lait de vache. 


\section{S u $\mathrm{m} \mathbf{m}$ a r y}

Studies were made on the electrophoretic composition of the sheep milk casein with different particles sizes. The results showed that the diameter of the particles does not influence the electrophoretic composition.

The ratio between the $\alpha_{s^{-}}$and $\chi$-casein from the sheep's milk casein is the same as that from the cows' $4 / 1$, while the ratios $\alpha: \beta$ and $\alpha: \beta: \gamma$ for the sheep' milk casein are different from those that are established for the cow's milk.

Reçu pour publication en avril 1971.

\section{Bibliographie}

[1] Annibaldi (S.) (1960), - Contribution à l'étude de la caséine. Le Latt 40, 593.

[2] BIER (M.) (1959). - Electrophoresis - Theory, Methods and Applications. Academic Press inc., New-York.

[3] Bloemendal (H.) (1963). - Zone Electrophoresis in Blocks and Columns. Elsevier Publishing comp., Amsterdam.

[4] Bohren (U. H.) and Wenner (R. V.) (1961). - Natural State of Milk Proteins. I. Composition of the Micellar and Soluble Casein of Milk after Centrifugal Sedimentation. J. Dairy Sci., 7, 1213.

[5] Caric (M.) and DJoRdjevic (J.). (1971). - The Microelectrophoretic Study of Casein. Milchwissenschaft, 26, 8, 487.

[6] Davidov (R. B.) et coll. (1966). - Issledovanie belkov moloka s promosciu elektronnoga mikroskopa. Molochn. promysl., 6, 16.

[7] FoRd (F. T.) and coll. (1955). - Composition of the Casein Containing Particles in Milk. J. Dairy Sci., 7, 843.

[8] Gebrauchsanweisung für den Mikroelektrophoreseapparat Kern LK 30.

[9] Hipp (N. J.) and coll. (1950). - Separation of $\gamma$ Casein. J. Amer. Chem. Soc., $72,4928$.

[10] Hipp (N. J.) and coll. (1952). - Separation of $\alpha, \beta$ and $\gamma$ Casein. J. Dairy Sci., 3, 272.

[11] Hostettler (H.) und ImнoF (K.) (1951). - Elektronenoptische Untersuchungen über den Feinbau der Milch. Milchwissenschaft, 10, 351.

[12] Payens (T. A. J.) (1966). - Associations of Caseins and their Possible Relation to Structure of the Casein Micelle. J. Dairy Sci., 11, 1317.

[13] SNOW (S. N.) and HAY (K. A.) (1966). - Variations in the Chemical Composition of Large and Small Casein Micelles. XVIII Inter. Dairy Congress, B 105.

[14] WAKe (G. R.) and BaLdwin (L. R.) (1961). - Analysis of Casein Fractions by Zone Electrophoresis in Concentrated Urea. Bioch. and Biophys. acta, 2,225 .

[15] WaUgh (D. F.) (1958). - The Interaction of $\alpha_{s}, \beta$ and $\chi$ Caseins in Micelle Formation. The Faraday Soc. Discussions, 25, 186.

[16] WebB (H. B). and Johnson (H. A.) (1965). - Fundamentals of Dairy Chemistry. The Avi Publish. Company inc. 